\title{
Development of Improved Devices for Handling and Restraining Experimental Laboratory Mice
}

Applied Biosafety:

Journal of ABSA International 2020, Vol. 25(I) 53-58

(C) ABSA International 2019

Article reuse guidelines:

sagepub.com/journals-permissions

DOI: $10.1177 / 1535676019892068$

journals.sagepub.com/home/apb

(S)AGE

\author{
Moshe Aftalion', David Gur', Noach Shalom², Yinon Levy', Avital Tidhar', \\ Yaron Vagima', Ayelet Zauberman', Tseela David ${ }^{3}$, Amir Rosner ${ }^{3}$, \\ Shay Weiss ${ }^{4}$, and Emanuelle Mamroud' ${ }^{1}$
}

\begin{abstract}
Introduction: Most animal handling procedures are associated with injuries among veterinary staff and laboratory animal researchers. However, much of the currently available animal handling equipment is inadequate, limiting access to the treated animal or making workflow cumbersome. Moreover, restraining animals to perform procedures, such as blood collection or injection, elicits stress in both the animal and the worker. Herein, we present 4 home-built restraint and blood collection devices in extensive use in our institute.

Methods: Animal laboratory workers and experienced veterinarians regularly using the devices $(n=14)$ were asked to complete a survey ranking the contribution of the devices to worker safety and procedural efficiency.

Results: The overwhelming majority of responders ( $\geq 75 \%)$ associated all 4 devices with substantial improvements in worker safety and procedural efficiency. There were no reports of impaired workflow or safety when using the devices.

Discussion: Infection and exposure control may be implemented on various levels, including use of safer procedures, such as injection and blood collection devices. The presented intuitive handling and restraint devices allow the animal worker/researcher to perform various procedures safely and efficiently while eliciting less animal and worker stress. The devices can be easily adjusted to accommodate animal size and disease status.
\end{abstract}

Conclusion: The current devices will serve as prototypes for design of devices for larger laboratory animals.

\section{Keywords}

restraint apparatuses, laboratory animals, safety, stress, bleeding, injection

\section{Introduction}

Laboratory-acquired infections (LAIs) have been documented since the early days of microbiological research. ${ }^{1}$ Laboratory animal workers face the risk of infection and exposure to hazardous agents that can adversely impact their health and safety. ${ }^{2}$ Occurrence is due to exposure to a wide variety of pathogens (ie, bacteria, viruses, fungi, and parasites) either by accident or following use of inappropriate technique, resulting in microbial penetration through inhalation, ingestion, contamination of skin and mucous membranes, or inoculation. ${ }^{1,3-5}$

For LAIs that resulted from laboratory accidents, it has been documented that approximately $25 \%$ to $30 \%$ of the incidents involved needle and syringe and $15 \%$ to $25 \%$ involved bites or scratches from animals. ${ }^{1,3}$ In a survey of 1625 field staff members of several federal veterinarian laboratories and services, it was observed that both laboratory and technical support staff were at risk from a variety of zoonotic infections, though generally to lesser extent than veterinary surgeons. ${ }^{6}$ In this survey, $71 \%$ of the veterinarians, $15 \%$ of the laboratory staff, and $42 \%$ of the technical staff reported injuries resulting from animal handling. ${ }^{6}$ Similarly, in a study involving 2532 veterinary school graduates, $64 \%$ reported 1 or more needlestick injuries in their career. ${ }^{7}$ Out of all 315 American Association of Zoo Veterinarian members that responded to a comprehensive occupational injuries survey, $61.5 \%$ reported on major

\footnotetext{
${ }^{\prime}$ Department of Biochemistry and Molecular Genetics, Israel Institute for Biological Research, Ness Ziona, Israel

${ }^{2}$ Department of Mechanics Design, Israel Institute for Biological Research, Ness Ziona, Israel

${ }^{3}$ Veterinary Center for Preclinical Research, Israel Institute for Biological Research, Ness Ziona, Israel

${ }^{4}$ Department of Infectious Diseases, Israel Institute for Biological Research, Ness Ziona, Israel
}

\section{Corresponding Authors:}

Moshe Aftalion and Emanuelle Mamroud, Department of Biochemistry and Molecular Genetics, Israel Institute for Biological Research, P.O. Box 19, Ness Ziona 74100, Israel.

Emails: moshea@iibr.gov.il; emmym@iibr.gov.il 


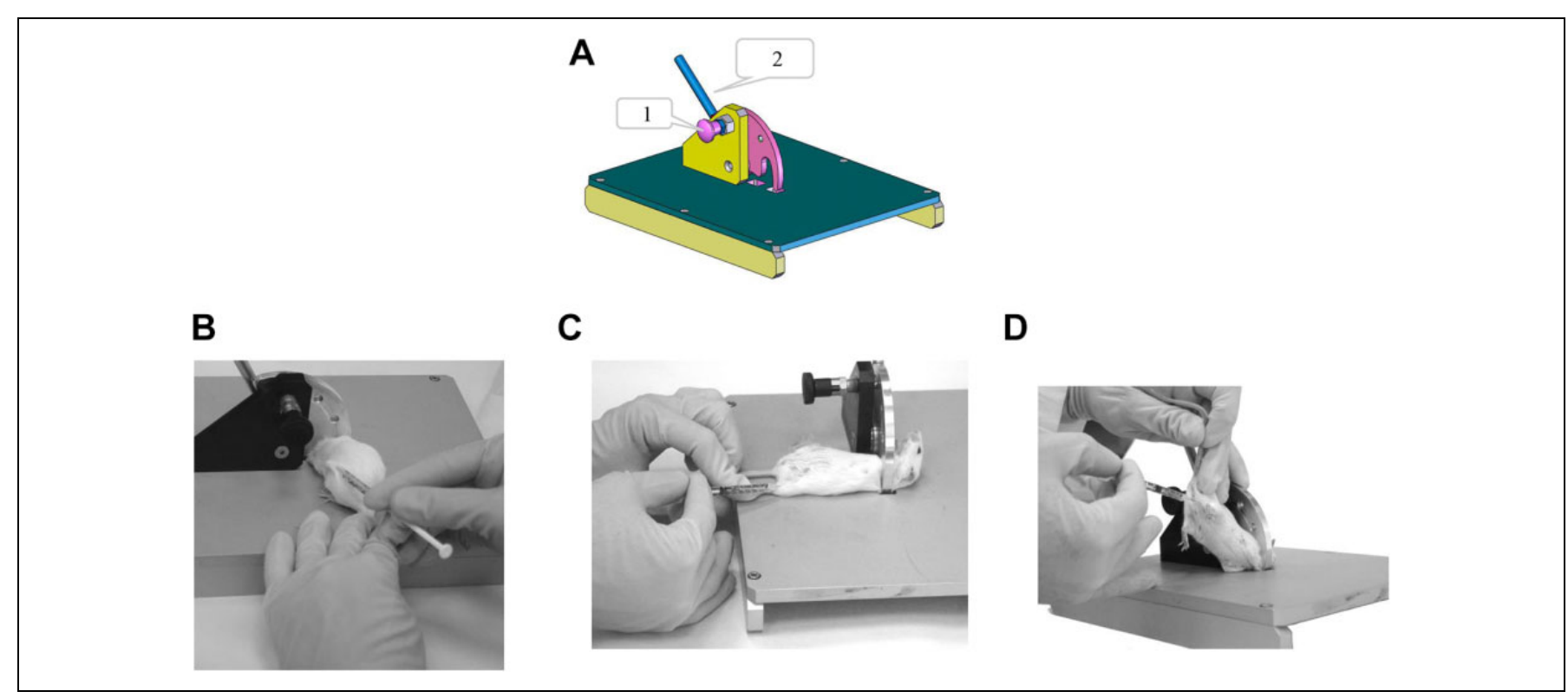

Figure I. Multipurpose restraint apparatus. (A) Schematic drawing of the device. I = locking knob; 2 = restraining lever. Device usage for (B) subcutaneous, $(C)$ intramuscular, and (D) intraperitoneal injections.

animal-related injury. ${ }^{8}$ Veterinary nurses reported a high rate of exposure to hazardous factors or conditions, with $98 \%$ suffering acute injuries in the form of animal bites/scratches and $71 \%$ sustaining at least 1 needlestick injury in the past. ${ }^{9}$ Needlestick or sharps injuries were reported by $75.3 \%$ of the 664 Australian veterinarians participating in a survey reviewing experiences in the preceding 12 months; $58.9 \%$ of survey responses involved a contaminated tool. ${ }^{10} \mathrm{~A}$ high exposure rate $(83.6 \%)$ was reported when handling small animals. Substantiating the concerns over the risks associated with needle handling in animal experimentation, a recently published case report described an event of vaccinia virus infection resulting from occupational needlestick injury. ${ }^{11}$

While a considerable percentage of incidents arise from failure to heed safety recommendations and guidelines, ${ }^{12,13}$ dedicated restraint devices can markedly improve worker safety. However, much of the currently available animal handling equipment is inadequate, limiting access to the treated animal or making workflow cumbersome. Chemical restraint, despite minimizing the risk of injury, is often unsuitable because it might change the course of the disease. In addition, the prevailing restraint and blood collection methods involve distressing procedures, such as cutting off the edge of the tail or capillary bleeding of the eye socket (retrobulbar venous sinus blood collection).

Herein, we describe new restraint and blood collection devices developed in the Israel Institute for Biological Research to suit mouse experiments within the framework of a BSL-3 research laboratory. The devices were carefully designed to increase worker safety and procedural efficiency while minimizing animal and worker discomfort and animal stress during handling. Devices were evaluated by experienced laboratory workers from the institute. Those workers were asked to complete a survey, with a separate questionnaire for each device, and reported a great satisfaction in terms of safety and efficiency of the procedures. Institutional veterinarians have approved the devices, and their use is routinely integrated in our institution in animal studies involving bacterial and viral pathogens. They were found to provide comfortable access to the entire animal body and obviate the need for coworker assistance.

\section{Methods}

\section{Animals}

This study was performed in accordance with the recommendations for the Care and Use of Laboratory Animals (National Institutes of Health $[\mathrm{NIH}]$ ) and Israeli law and were approved by the Institutional Ethics Committee for Animal Experiments (protocols M-20-16, M-14-16).

\section{Improved Restraint and Blood Collection Devices}

Multipurpose restraint apparatus (Figure I). This device is composed of a semicircular restraint plate with a small rounded indentation at one edge, which is simply lowered over the mouse's neck or upper back and then locked in place (see Figure 1 and "User's Guide" sections). It provides for animal restraint at the neck/chest while maintaining maximal access to various parts of the body. At the same time, it limits animal neck motion and allows access to the posterior part of its body. Moreover, the diameter of the restraint plate can be adjusted to accommodate very small, large, or diseased animals. The multipurpose restraint apparatus enables safe and simple injections to mice via the subcutaneous, intramuscular, intradermal, and intraperitoneal routes. Unlike common practices, intradermal injections performed with this device do not require animal sedation. 


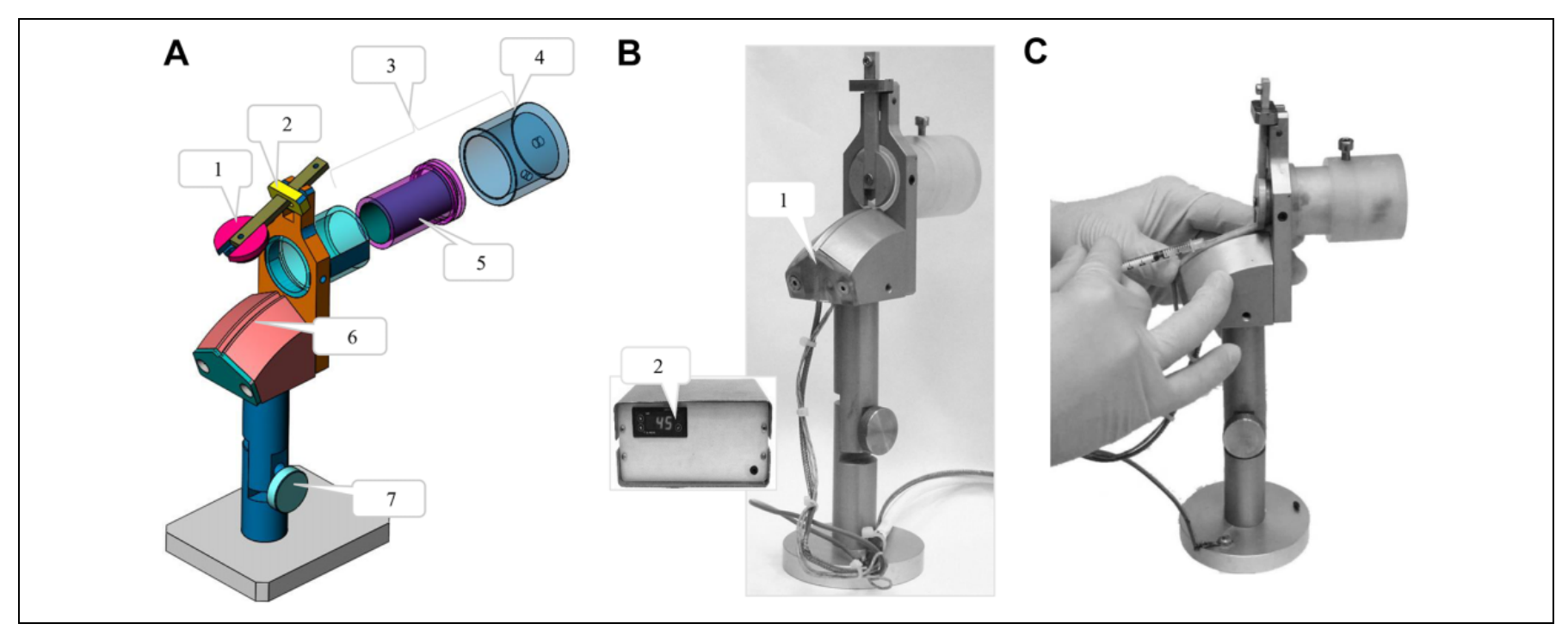

Figure 2. Intravenous tail-vein injection and blood collection apparatus. (A) Schematic drawing of the device. I = compartment lock; $2=$ locking ring; 3 = adjustable cabin; $4=$ adjustable cylinder for large mice; $5=$ optional inner adjustable cylinder for small mice; $6=$ tail holder channel; 7 = angle setting. (B) The device and the thermal controller. I = inner thermal sensor and heating element; $2=$ temperature setting and display. (C) Device usage for intravenous tail-vein injection.

User guide. Pull out the locking button and turn $90^{\circ}$ in either direction to maintain the restraining lever in an open state. Then, lower the restraining lever to the left side until it touches the metal working surface. Hold the mouse's tail with your right hand and gently drag the mouse backward toward the restraint plate. With your left hand, hold the tip of the restraint lever and lift it gently (without placing too much pressure on the mouse) until the mouse is securely restrained. For ventral access, grasp the mouse at its trunk and roll it onto its back while reducing the restraint by slightly lowering the restraint lever with your left hand. Release the mouse and lock the restraint plate by turning the locking button $90^{\circ}$ in either direction. Now the mouse is restrained and ready for the procedure. To release the mouse, hold its tail with your right hand, unlock the button with your left hand, and lower the restraint lever.

Intravenous tail vein injection and blood collection apparatus (Figure 2). This device is specially designed for intravenous injection. The mouse is restrained within a closed, cylindrical restraining tube, with only the tail exposed and held in a dedicated groove. There, the tail can be locally heated throughout the duration of the experiment, avoiding the need to heat the entire animal body, thereby minimizing animal stress as well as undesirable physiological responses. In addition, the device enables controlled and swift blood collection from the tail vein. The device is equipped with a restraining tube adaptor to adjust the diameter for smaller mice and longer restraining tubes to accommodate longer animals. In addition, the external tail rest can be adjusted to suit long tails, reducing risk of needle pricks. The apparatus design demands less worker specialization to obtain the desired result.

User guide. Switch on the heating system and set the desired temperature (recommended temperature range between $40^{\circ}$ and $42^{\circ} \mathrm{C} / 104^{\circ}$ and $107.6^{\circ} \mathrm{F}$ ). After reaching the desired temperature, lift the locking ring and open the compartment lock. Hold the mouse by its tail and allow it to enter the restraining tube head first (in some cases, a more firm hold is needed: hold the mouse both tail and scruff of the neck and lead it head first into the restraining tube). Release the lid while passing the tail through the designated aperture in the lid. The locking ring will automatically close and prevent accidental opening. Insert the tail into the groove in the tail holder, disinfect the injection site with $70 \%$ alcohol, and wait approximately 1 minute until the tail is heated. Inject/bleed as desired. To release the mouse, hold the tail with your hand and lift the locking ring with your other hand to open the compartment lock. Gently withdraw the mouse backward, out of the restraining tube.

Tail blood collection device (Figure 3). This apparatus was designed to enable safe and accurate transverse tail incision followed by blood collection. The tail is passed between a 22 surgical blade and a protective plate positioned just above it. The height of the blade can be adjusted to control depth of incision. In addition, there are holders for both a microtainer test tube and $1.5 \mathrm{~mL}$ test tubes for whole-blood sampling. The setup supports a safe and rapid workflow for safe and accurate transverse tail incisions and blood collection. The controlled procedure allows repeated blood collection and minimizes tail injury.

User guide. Carefully attach the blade and adjust the height to suit the size of the mouse being handled and depth of incision. Note that the blade should not protrude beyond $2 \mathrm{~mm}$. Prepare the necessary test tube(s) and label clearly. Place the test tube(s) in the designated holder. It is recommended to warm the animal prior to bleeding in a warming cabinet (body temperature for up to 10 minutes) to dilate the peripheral blood vessels. Disinfect the tail with an antimicrobial solution such as 


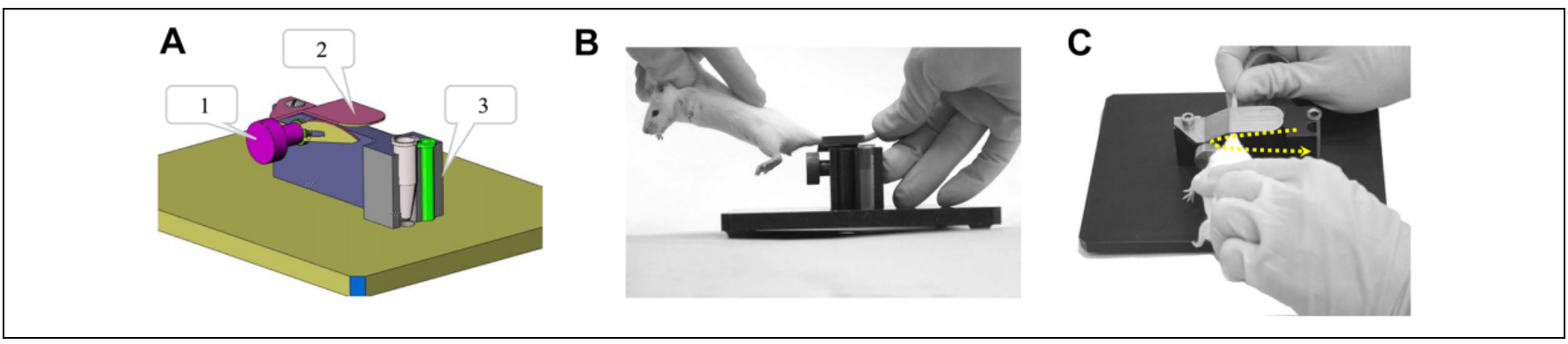

Figure 3. Tail blood collection device. (A) Schematic drawing of the device. I = blade angle setting; 2 = blade cover; $3=$ tube holder. (B, C) Device usage for tail-vein blood collection. The yellow dotted arrow indicates that the animal tail, held perpendicular to the blade, is inserted from the upper portion of the blade cavity and following incision of the vein, is excised from its lower part.

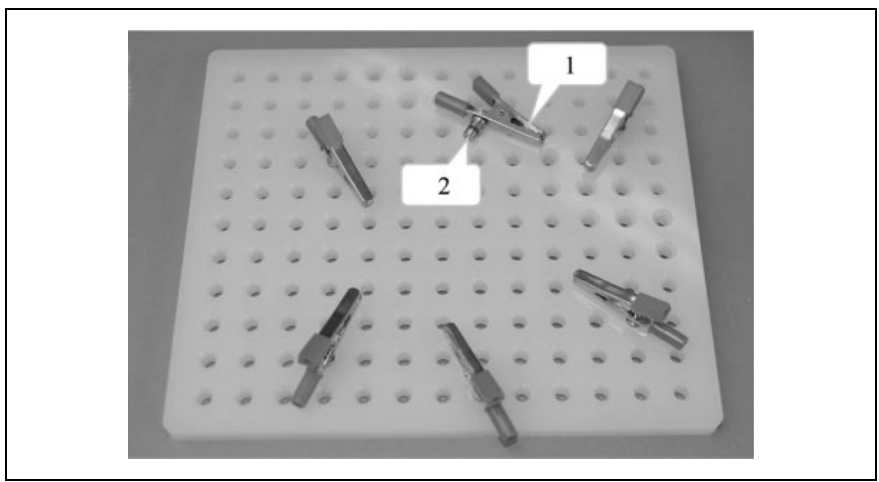

Figure 4. Surgical perforated platform with adjustable pincer $(I)$ and peg (2) to anchor to the board.

$70 \%$ alcohol. Grasp the scruff of the neck with one hand and the tip of the tail with the other hand (Figure 3B). While holding the tail taut, press the tail against the blade in a sort of sideways slicing motion to cause laceration on the tail (Figure 3C). Allow the blood to drip into the appropriate test tube. Apply pressure on the incision site with a sterile dry gauze pad until the bleeding stops. Be sure to make the incision as close as possible to the tip of the tail while accounting for the required volume of blood, the location and number of scars from previous blood collection events, and animal health status.

Surgical platform (Figure 4). This apparatus is composed of a perforated board and attachable pincers (alligator clips with attached pegs inserted into a costume pegboard), allowing safe and flexible surgery procedures and therefore eliminating the need for sharp pins and peg boards.

User guide. Place the anesthetized animal (for surgery) or corpse (for necropsy) on the perforated platform in the desired position. Fix it into place by using the attachable pincers grip (spring-loaded clips with attached pegs). Change pincers' position as desired by moving them to the appropriate hole. Add attachable pincers as needed. To avoid possible tissue damage when restraining anesthetized animals, wrap the mouse limb with gauze pad.
Note: All apparatuses can be disinfected with ethanol, iodine solutions, bleach (500 ppm, for 10 minutes), followed by rinsing with tap water or alternatively by formalin vapor.

\section{User Satisfaction Survey}

Veterinarians and laboratory animal workers in our institution with at least 10 years of animal handling experience were asked to complete a short survey assessing the contribution of the devices to worker safety and procedural efficiency after using each one several times. Assessment was based on a 4-category scale: interferes, no effect, improvement, and significant improvement.

\section{Results}

\section{User Satisfaction Survey}

In total, 14 laboratory workers from a variety of animal research laboratories in our institution, with experience working with all 4 described restraint devices, completed the survey, with a separate questionnaire completed for each device. All of the workers reported that the multipurpose restraint apparatus contributed to their safety (Figure 5A), with $83 \%$ indicating that they believed there was substantial improvement in their safety while using it. In addition, $92 \%$ of the users indicated that using the device contributed to the efficiency of the procedure; $59 \%$ gave the highest score (significantly improved), while $8 \%$ opined that using the device did not change the productivity of animal laboratory procedures. Most importantly, none of the users thought that using the device impaired technical performance. Similar feedback was provided with regards to the intravenous tail vein injection apparatus (Figure 2). All responders believed that the device contributed to their safety, with $86 \%$ reporting on a significant contribution (Figure 5B). In addition, most of the users (93\%) opined that the device improved procedural efficiency, with $77 \%$ reporting on a significant contribution to efficiency. Again, none of the users reported that the device reduced the safety or efficiency of the procedure. The tail vein blood collection apparatus (Figure 3) also received very positive reviews; all the workers found the device to be very helpful in improving user safety and procedural efficiency. In using the surgical platform 


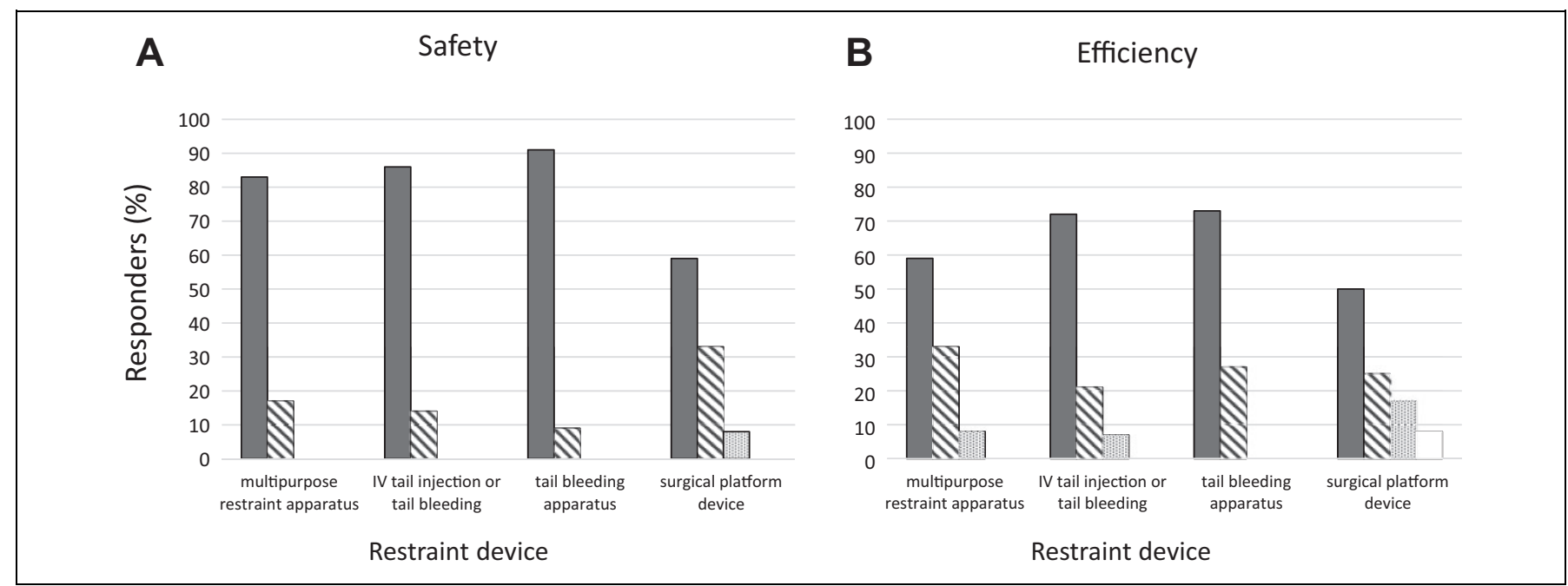

Figure 5. User satisfaction results with the various restraint and blood collection devices $(n=14)$ on the contribution of the handling and restraint devices to $(A)$ user safety and $(B)$ procedural efficiency. Filled bars = significant improvement; diagonal-striped bars = improvement; dotted bars = no effect; empty bars $=$ interferes.

device, $92 \%$ of the workers reported that it contributed to worker safety, and $75 \%$ claimed that the efficiency of the procedure was improved when using the device.

In summary, the vast majority of survey participants found that the handling and restraint devices provided a valuable contribution to both user safety and procedural efficiency.

\section{Discussion}

Because laboratory animal researchers and veterinary staff engage in high-risk animal handling procedures, it is not surprising that injuries are relatively common, albeit significantly underreported. ${ }^{2,14}$ While most injuries were associated with minor sequelae, they could come along with severe and serious consequences. ${ }^{15,16}$ Recently, a survey of LAIs in BSL-3 and BSL-4 laboratories implied that $14 \%$ of the incidents were due to accidents while conducting procedures in animal experiments and performing animal care in high contaminant BSL laboratories. ${ }^{17}$ Although personal protective equipment is highly effective in preventing accidental infection through inhalation, ingestion, and mucosal splashes, accidents involving bites, scratches, and stabbing from infected animals or contaminated sharp objects are directly bypassing the protective gear and might result in life-threatening illness. ${ }^{18}$ Infection and exposure control may be implemented on various levels, including use of safer injection and blood collection devices. In addition, performing the procedures using currently available commercial devices are stressful and might involve animal suffering and poor well-being. The presented handling and restraint devices are in extensive use in our institute and have significantly improved worker safety and access to all parts of the animal body. The reports from our institute researchers indicated marked improvement in procedural efficiency and safety. The presented apparatuses can serve as prototypes for design of devices for additional laboratory animals. Indeed, following the successful implementation of these mouse restraint devices, a similar project for development of restraint apparatuses for rats is now under development in our institution. In addition, those apparatuses might improve animal welfare and minimize distress of the worker, issues that require further comparative research/follow-up study.

\section{Conclusion}

Restraint devices should be suitable in size, design, and operation to minimize discomfort, pain, distress, and the potential for injury to the animal and the research stuff. ${ }^{19}$ The handling and restraint apparatuses presented here improved worker safety and access. The devices were easily adjusted to accommodate animals of variable size and disease statuses. The devices were simple to use and maintain and could be easily self-manufactured, and they enhanced animal laboratory safety and procedural efficiency. The current devices will serve as prototypes for design of devices for additional laboratory animals.

\section{Ethical Approval Statement}

This study was performed in accordance with the recommendations for the Care and Use of Laboratory Animals (National Institutes of Health $[\mathrm{NIH}])$ and Israeli law and were approved by the Institutional Ethics Committee for Animal Experiments (protocols M-20-16, M14-16).

\section{Statement of Human and Animal Rights}

Not applicable to this study.

\section{Statement of Informed Consent}

Not applicable to this study. 


\section{Declaration of Conflicting Interests}

The authors declared no potential conflicts of interest with respect to the research, authorship, and/or publication of this article.

\section{Funding}

The authors disclosed receipt of the following financial support for the research, authorship, and/or publication of this article: We thank the Israel Institute for Biological Research for funding this project. Grant No. SB/5112/182.

\section{ORCID iD}

Emanuelle Mamroud (D) https://orcid.org/0000-0001-5685-293X

\section{References}

1. Pike RM. Laboratory-associated infections: incidence, fatalities, causes and prevention. Ann Rev Microbiol. 1979;33:41-66.

2. Weigler BJ, Di Giacomo RF, Alexander S. A national survey of laboratory animal workers concerning occupational risks for zoonotic diseases. Comp Med. 2005;55(2):183-191.

3. Byers KB, Harding AL. Laboratory-associated infections. In: Wooley D, Byers KB, eds. Biological Safety: Principles and Practice. 5th ed. Washington, DC: ASM Press, Inc; 2017:59-62.

4. Singh K. Laboratory acquired infections. Clin Infect Dis. 2009; 49(1):142-147.

5. Sewell DL. Laboratory associated infections and biosafety. Clin Microbiol Rev. 1995;8(3):389-405.

6. Constable PJ, Harrington JM. Risks of zoonoses in a veterinary service. Br Med J (Clin Res Ed). 1982;284(6311):246-248.

7. Wilkins JR, Bowman ME. Needlestick injuries among female veterinarians: frequency, syringe contents and side-effects. Occup Med (Lond). 1997;47(8):451-457.

8. Hill DJ, Langley RL, Morrow WM. Occupational injuries and illnesses reported by zoo veterinarians in the United States. $J$ Zoo Wildl Med. 1998;29(4):371-385.
9. Van Soest EM, Fritschi L. Occupational health risks in veterinary nursing: an exploratory study. Aust Vet J. 2004;82(6):346-350.

10. Leggat PA, Smith DR, Speare R. Exposure rate of needlestick and sharps injuries among Australian veterinarians. J Occup Med Toxicol. 2009;4:25.

11. Whitehouse ER, Rao AK, Yu YC, et al. Novel treatment of a vaccinia virus infection from an occupational needlestick-San Diego, California, 2019. MMWR Morb Mortal Wkly Rep. 2019; 68(42):943-946.

12. Weese JS, Faires MA. Survey of needle handling practices and needlestick injuries in veterinary technicians. Can Vet J. 2009; 50(12):1278-1282.

13. Weese JS, Jack DC. Needlestick injuries in veterinary medicine. Can Vet J. 2008;49(8):780-784.

14. Voide C, Darling KE, Kenfak-Foguena A, Erard V, Cavassini M, Lazor-Blanchet C. Underreporting of needlestick and sharps injuries among healthcare workers in a Swiss University Hospital. Swiss Med Wkly. 2012;142:w13523.

15. Veenhuizen MF, Wright TJ, McManus RF, Owens JG. Analysis of reports of human exposure to Micotil 300 (tilmicosin injection). J Am Vet Med Assoc. 2006;229(11):1737-1742.

16. Ashford DA, di Pietra J, Lingappa J, et al. Adverse events in humans associated with accidental exposure to the livestock brucellosis vaccine RB51. Vaccine. 2004;22(25-26): 3435-3439.

17. Wurtz N, Papa A, Hukic M, et al. Survey of laboratory-acquired infections around the world in biosafety level 3 and 4 laboratories. Eur J Clin Microbiol. 2016;35(8):1247-1258.

18. Gunther S, Feldman H, Geisbert TW, et al. Management of accidental exposure to Ebola virus in biosafety level 4 laboratory Hamburg Germany. J Infect Dis. 2011;204:S785-S790.

19. National Research Councio. Guide for the Care and Use of Laboratory Animals. 8th ed. Washington, DC: The National Academies Press; 2011. 